\title{
Outcome and patients' satisfaction after functional treatment of acute lateral ankle injuries at emergency departments versus family doctor offices

\author{
Patrik R Schwab², Lorin M Benneker*1, Stefan Eggli1, Heinz Zimmermann² \\ and Aristomenis K Exadaktylos ${ }^{2}$
}

\begin{abstract}
Address: ${ }^{1}$ Department for Orthopaedic Surgery, Inselspital, University of Berne, Berne, Switzerland and ${ }^{2}$ Emergency Unit, Inselspital, University of Berne, Berne, Switzerland

Email: Patrik R Schwab - patrik.schwab@gmx.ch; Lorin M Benneker* - lorin.benneker@insel.ch; Stefan Eggli - stefan.eggli@insel.ch; Heinz Zimmermann - heinz.zimmermann@insel.ch; Aristomenis K Exadaktylos - exadaktylos@exadaktylos.ch

* Corresponding author
\end{abstract}

Published: 23 December 2008

BMC Family Practice 2008, 9:69 doi:10.1 186/1471-2296-9-69
Received: 20 April 2008

Accepted: 23 December 2008

This article is available from: http://www.biomedcentral.com/I47/-2296/9/69

(c) 2008 Schwab et al; licensee BioMed Central Ltd.

This is an Open Access article distributed under the terms of the Creative Commons Attribution License (http://creativecommons.org/licenses/by/2.0), which permits unrestricted use, distribution, and reproduction in any medium, provided the original work is properly cited.

\begin{abstract}
Background: In some Western countries, more and more patients seek initial treatment even for minor injuries at emergency units of hospitals. The initial evaluation and treatment as well as aftercare of these patients require large amounts of personnel and logistical resources, which are limited and costly, especially if compared to treatment by a general practitioner. In this study, we investigated whether outsourcing from our level I trauma center to a general practitioner has an influence on patient satisfaction and compliance.
\end{abstract}

Methods: This prospective, randomized study, included $\mathrm{n}=100$ patients who suffered from a lateral ankle ligament injury grade I-II $(16,17)$. After radiological exclusion of osseous lesions, the patients received early functional treatment and were shown physical therapy exercises to be done at home, without immobilization or the use of stabilizing ortheses. The patients were randomly assigned into two groups of 50 patients each: Group A (ER): Follow-up and final examination in the hospital's emergency unit. Group B (GP): Follow-up by general practitioner, final examination at hospital's emergency unit. The patients were surveyed regarding their satisfaction with the treatment and outcome of the treatment.

Results: Female and male patients were equally represented in both groups. The age of the patients ranged from $16-64$ years, with a mean age of 34 years (ER) and 35 years (GP). 98\% ( $n=$ 98) of all patients were satisfied with their treatment, and $93 \%(n=93)$ were satisfied with the outcome. For these parameters no significant difference between the two groups could be noted $(p=0.7406$ and 0.7631 respectively). $39 \%$ of all patients acquired stabilizing ortheses like ankle braces (Aircast, Malleoloc etc.) on their own initiative. There was a not significant tendency for more self-acquired ortheses in the group treated by general practicioners $(p=0,2669)$.

Conclusion: Patients who first present at the ER with a lateral ankle ligament injury grade I-II can be referred to a general practitioner for follow-up treatment without affecting patient satisfaction regarding treatment and treatment outcome. 


\section{Background}

In some Western countries an increasing number of patients seeks help directly at emergency department without seeing their general practitioner first even for minor complaints as inversion trauma of the ankle [1] The occurrence of these acute lateral ankle ligament injuries is estimated at 1:10'000 people per day with a major impact on health care costs [2,3]. This number translates into approximately 650 lateral ankle ligament injuries per day in Switzerland, with a high incidence rate for certain sports [4-6]. In a Scandinavian study acute lateral ankle ligament injuries accounted for $7-10 \%$ of all ER admittances, which makes these injuries one of the primary reason for admittance to the emergency room in those countries [7]. Most of the patients are younger than 35 years, with the broadest representation of patients between the age of 15-19 years and an elevated risk especially for young female athletes $[4,8,9]$.

After having eliminated the possibility of a fracture, early functional treatment is the therapy of choice for mild and acute forms of lateral ankle ligament injuries since it there are no surgery related risks as infections, thrombosis, embolism, scars, pain, wound healing problems or anesthesia related adverse effects. The period of recovery has shown to be shorter in non-operative treatment with the same long-term functional results and joint stability [10-17]. From a socio-economic standpoint, this kind of therapy is clearly preferable to other forms of therapy, in particular surgery, but also immobilization $[14,15,18]$. There is no strong evidence which functional treatment is best suited for these grade I-II lateral ankle ligament injuries [19] Contrary to recommendations of other authors, in our institution we only apply an elastic bandage to prevent swelling in initial phase even for grade III injuries and do not generally advocate the use of stabilizing semi rigid ortheses for the non athletic population [5,18-20].

With regard to bring down costs produced by minor midfoot and ankle injuries our unit already developed the 'Bernese Ankle Rules' which are based on the original 'Ottawa Ankle Rules' and could significantly enhance the specifity for detecting fractures of the ankle and lowering the amount of unnecessarily obtained radiographs [21]. Aside of the initial diagnosis and treatment also follow-up treatment of patients in emergency units requires large amounts of personnel and logistical resources, and is much costlier than treatment by a general practitioner $[22,23]$. The goal is therefore to refer these patients as quickly as possible to their general practitioner for followup treatment. The question that arose was whether the patients who were admitted to the emergency room of the University Hospital with a mild lateral ankle ligament injury reported reduced satisfaction with regards to their therapy as well as the treatment outcome if they are referred to their general practitioner for follow-up care. Objective of this study was to investigate the influence of similar aftercare under different settings (ER vs GP) on patients satisfaction after minor trauma as ankle sprains.

\section{Methods}

100 consecutive patients who were admitted to our trauma center between May and October 2004 with lateral ankle ligament injuries of all grades were included in this prospective randomized study. Patients agreed to participate befor randomization. The protocol was presented to the ethical committee of the University of Bern but no formal approval was necessary as the study was classified as quality control investigation of an established treatment.

Osseous lesions were excluded by clinical examination according to the 'Bernese ankle rules' and by additional antero-posterior and lateral radiographs [21]. The exclusion criteria were: non-obvious supination trauma, previous treatment by a general practitioner or in a different clinic, rupture of deltoid ligament, fractures, and refusal to participate on the part of the patient.

Regardless of the grade of the injury the patients received an early functional therapy, consisting of early antiphlogistic measures according the RICE procedure (Rest - Ice - Compression - Elevation), elastic support bandage, early return to full weight bearing after only a short period of immobilization and instruction for motion exercises to be begun at home after the acute phase. These early active and passive joint movements promote healing and prevent edema [24]. The physical therapy exercises were developed in collaboration with the Swiss Sports Institute (Eidgenössische Turn- und Sportschule in Magglingen, ESSM) and are designed to strengthen the peroneal muscles and improve neuromuscular control through proprioceptive training $[16,25]$. Most patients were confident with an instructional handout for range of motion, weight bearing and neuromuscular training exercises; only a small percentage (less than 10\%) demanded physiotherapeutic guidance. All patients were informed that we do not recommend the use of a semi-rigid ankle ortheses.

Patients then were randomly assigned into two groups: Group ER: emergency room ( $n=50,22$ males, 28 female, average age $=34$ years ; Follow-up within one week in our clinic. Group GP: general practitioner $(\mathrm{n}=50,25$ male, 25 female, average age: 35 years); Follow-up within one week at the general practitioner's office. Both groups had a final control in our clinic 2 months after their trauma when the patients were asked to fill a questionnaire for evaluation of subjective satisfaction regarding treatment management and outcome (Table 1). On this protocol there was room left for additional information as the independent acquisition of stabilizing ortheses or the like. At final con- 
Table I: Questionnaire for evaluation of subjective satisfaction regarding therapy and treatment outcome.

Satisfaction regarding therapy and treatment outcome

Please define your level of satisfaction regarding treatment and therapy:

very satisfied satisfied dissatisfied disappointed very disappointed

If dissatisfied or disapointed please specify:

Please define your level of satisfaction regarding treatment outcome:

very satisfied satisfied dissatisfied disappointed very disappointed

If dissatisfied or disappointed please specify:

Did you acquire a stabilizing ortheses independently?

Additional remarks:

trol the patients underwent a standardized physical examination for objective measure (Table 2). No stress radiographs were performed at any time.

Statistical analysis then was performed to evaluate the dependence of patients' satisfaction on where the followup was carried out by using a Chi-squared and Fishers' exact test with a significance level set at $\mathrm{p}<0,05$.

\section{Results}

The final check-up for all patients took place 2 months after the trauma ( $\mathrm{n}=97,2$ patients died ( 1 suicide/ 1 of natural causes), one patient moved away without leaving a forwarding address).

Satisfaction rate regarding therapy two months after the trauma was high for both groups: in the ER group, 18 patients $(37 \%)$ were very satisfied, 28 patients $(57 \%)$ were satisfied, three patients $(6 \%)$ were dissatisfied. In the GP group, 16 patients (33\%) were very satisfied, 31 patients $(65 \%)$ were satisfied and one patient (2\%) was dissatisfied (fig. 1). The main reason for dissatisfaction was a feeling of being under-treated. The differences between the two groups are not significant $(\mathrm{p}=0.7406)$. In both

Table 2: Protocol and results of objective examination at follow up one week (only group ER) and two months after lateral ankle injury (both groups).

\begin{tabular}{|c|c|c|c|c|c|c|c|}
\hline & & \multicolumn{2}{|c|}{ ER I wk } & \multicolumn{2}{|c|}{ ER $2 \mathrm{mts}$} & \multicolumn{2}{|c|}{ GP $2 \mathrm{mts}$} \\
\hline & & [no] & [\%] & [no] & [\%] & [no] & [\%] \\
\hline \multirow[t]{2}{*}{ swelling and/or haematoma } & malleolus lateralis & 34 & 70.4 & 11 & 22.5 & 14 & 29.2 \\
\hline & malleolus medialis & 2 & 4.1 & 2 & 4.1 & 2 & 4.2 \\
\hline \multirow[t]{6}{*}{ pain at palpation of: } & capitulum fibulae & 0 & 0 & 0 & 0 & 0 & 0 \\
\hline & malleolus lateralis & 8 & 16.3 & 0 & 0 & 0 & 0 \\
\hline & malleolus medialis & 4 & 8.2 & 0 & 0 & 0 & 0 \\
\hline & lig talofibulare anterius & 35 & 71.4 & 20 & 40.1 & 18 & 37.5 \\
\hline & metatarsalia & 1 & 2 & 0 & 0 & 0 & 0 \\
\hline & anterior syndesmosis & 14 & 28.6 & I & 2 & 0 & 0 \\
\hline
\end{tabular}

[number of patients/percentage] 


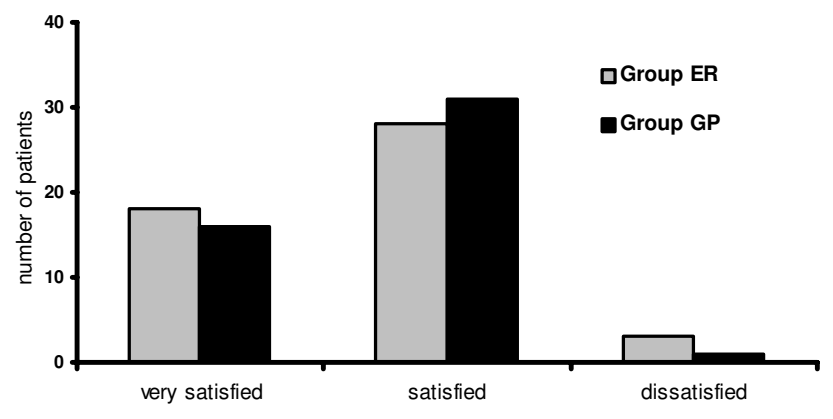

Figure I

Subjective satisfaction with regard to treatment of patients $\mathbf{2}$ months after acute lateral ligament injury. Aftercare conducted by emergency room physicians (group ER) or general practitioners (group GP).

groups no patients were found to be disappointed or very disappointed.

Satisfaction regarding treatment outcome was evaluated two months after the trauma: In the ER group, 18 patients (37\%) were very satisfied, 27 patients (55\%) were satisfied and 4 patients (8\%) were dissatisfied. The main reason for dissatisfaction was persistent swelling and pain. In the GP group, 15 patients (31\%) were very satisfied, 30 patients $(63 \%)$ were satisfied and 3 patients $(6 \%)$ were dissatisfied. No patients were disappointed or very disappointed. The uniform reason for dissatisfaction in this group was persistent pain (fig. 2). Again the differences between the two groups were not significant $(\mathrm{p}=0.7631)$.

36 out of a total of 97 patients (15 in ER group/21 in GP group) acquired an ankle brace (mostly Aircast ${ }^{\circledR}$ (Summit, NJ USA) or Malleoloc ${ }^{\circledast}$ (Bauerfeind AG, Zeulenroda, Ger-

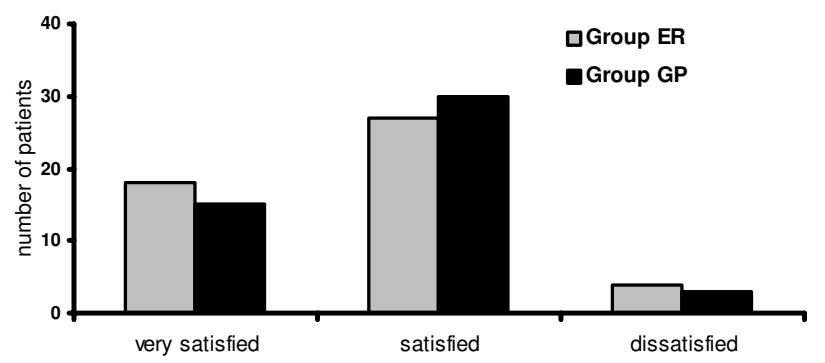

Figure 2

Subjective satisfaction with regard treatment outcome of patients $\mathbf{2}$ months after acute lateral ligament injury that received an early functional treatment. Aftercare conducted by emergency room physicians (group ER) or general practitioners (group GP). many)), i.e. $39 \%$ of all patients. There was a tendency for more self-acquired ortheses in the GP group although there was no significant correlation to where the treatment took place or the level of satisfaction $(p=0.129) .6$ patients in the ER group and 3 patients in the GP group required physiotherapy $(\mathrm{p}=0.253)$ (Table 3$)$.

At 2 months after trauma at the objective physical examination swelling and/or haematoma above the lateral malleolus was present in 11 patients (22\%) in the ER group and 14 patients (29\%) in the GP group. Swelling and/or haematoma above the medial malleolus were diagnosed in 2 patients in each group ( $4 \%$ in each group). Pain with pressure above talofibular ligamentswas only diagnosed in 20 patients (40\%) in the ER group and in 18 patients (38\%) in the GP group. In the ER group, 1 patient (2\%) complained of persistent pain with pressure in the area of the syndesmosis. Pain with pressure was not reported for any of the other pressure areas (table 2, fig. 3).

Objective collection of data could again not find significant differences between the two groups.

\section{Discussion}

At our trauma center, acute lateral ankle ligament injuries of all grades are addressed with early functional treatment. The initial treatment is based on the RICE method (Rest Ice - Compression - Elevation) in conjunction with the use of crutches, followed by physical therapy exercises which the patient can do independently at home. These early active and passive joint movements prevent edema, are designed to strengthen the peroneal muscles and improve neuromuscular control through proprioceptive training [16,24-26].

The analgesics that are prescribed are limited to paracetamol, mefenamic acid and/or Cox-2 inhibitors. Taking non-steroidal anti-inflammatory drugs has no effect on the healing process [24].

The basis of our study was the question whether patients, who chose the emergency room of the University Hospital for initial diagnosis, are satisfied with our initial therapy, and whether there is a difference in the level of satisfaction

Table 3: Overview of number/percentage of patients who needed physiotherapeutic assistance or bought an ankle ortheses independently.

\begin{tabular}{ccc}
\hline & Ankle brace & Physiotherapy \\
\hline Group ER $(n=49)$ & $15(30.6 \%)$ & $6(12.2 \%)$ \\
\hline Group GP $(n=48)$ & $21(43,8 \%)$ & $3(6.3 \%)$
\end{tabular}




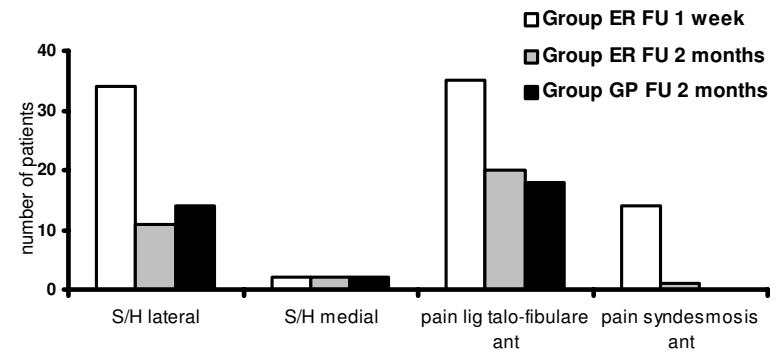

Figure 3

Objective measure of treatment outcome one week (group ER) and two months (both groups) after acute lateral ligament injury. Aftercare conducted by emergency room physicians (group ER) or general practitioners (group GP) [S/H = swelling and/or haematoma.

between the patients who receive follow-up care at our clinic or at their general practitioner's office. Since our clinic does not prescribe ankle braces (Malleoloc or Aircast), the additional question arose as to whether the patients acquired such stabilizing ortheses themselves or were prescribed ankle braces by their general practitioner during the follow-up visit.

The results confirm that the method of follow-up care has neither influence on either patient satisfaction nor the subjective or objective treatment outcome. It is encouraging that the results show that patients are satisfied with the early functional treatment and don't feel as if they were under-treated. Follow-up care can be transferred to general practitioners without affecting patient satisfaction. This leads to a significant decrease in the costs for followup care. These results are consistent with other investigations that report a high satisfaction rates at lower costs for patients with minor injuries when treated by their chosen family physician $[22,23,27]$.

The fact that $39 \%$ of all patients independently acquired stabilizing orthoses such as Aircast, Malleoloc etc. presents the challenge of patient education for the doctor making the initial diagnosis. Although there was a slight tendency for more self-acquired ortheses in the second group (GP) there was no significant difference. Use of stabilizing orthoses has no influence on the treatment outcome at two months follow-up, though the patient's comfort level may be improved through external stabilization and studies report a faster return to work [19]. However, this increased comfort level comes with a high price tag: with an incidence rate of 1 trauma/10.000/day [2,3], . the average number of acute lateral ankle ligament injury cases per year in Switzerland would be approx. 240'000. $39 \%$ of the patients, i.e. 93 '000 patients per year, acquired stabilizing ortheses, at an average cost of CHF 100, which amounts to a total amount of additional costs of approx. CHF 9.3 million per year.

The reasons for acquiring auxiliary devices are varied. The two most common answers given were increased comfort and the fact that friends/family members were treated with stabilizing ortheses.

\section{Conclusion}

Our patients with lateral ankle injuries receiving early functional treatment report a high satisfaction rate (Additional file 1). Patient satisfaction does not depend on where follow-up care is performed (emergency room vs. general practitioner). Subsequently, patients can be referred to their general practitioner for follow-up care. This can ease the strain on resources at the emergency units of hospitals and leads to significant cost savings and continous physician-patient relationship.

Independent acquisition of orthopedic auxiliary devices (Aircast, Malleoloc etc.) is not significantly different between the two groups, yet amounts to the considerable number of $39 \%$ and additional costs.

\section{Competing interests}

The authors declare that they have no competing interests.

\section{Authors' contributions}

PS did the clinical trial and participated in the design of the study and the statistical analysis. LB paticipated in the design of the study and controlled the use of the bernese ankle rules. SE patricipated in the design of the study. HZ did the one part of the statistical analysis. AE participated in the design of the study and performed the other statistics. All Authors read and approved the final manuscript.

\section{Additional material}

\section{Additional File 1}

Flowchart. Outcome of lateral ankle injuries.

Click here for file

[http://www.biomedcentral.com/content/supplementary/1471-

2296-9-69-S1.ppt]

\section{References}

I. Rubin MA, Bonnin MJ: Utilization of the emergency department by patients with minor complaints. J Emerg Med 1995 , I 3:839-842.

2. McCulloch PG, Holden P, Robson DJ, Rowley DI, Norris SH: The value of mobilisation and non-steroidal anti-inflammatory analgesia in the management of inversion injuries of the ankle. Br J Clin Pract 1985, 39:69-72.

3. Brooks SC, Potter BT, Rainey JB: Treatment for partial tears of the lateral ligament of the ankle: a prospective trial. $\mathrm{Br} M e d \mathrm{~J}$ (Clin Res Ed) I98I, 282:606-607. 
4. Hosea TM, Carey CC, Harrer MF: The gender issue: epidemiology of ankle injuries in athletes who participate in basketball. Clin Orthop Relat Res 2000:45-49.

5. Boyce SH, Quigley MA, Campbell S: Management of ankle sprains: a randomised controlled trial of the treatment of inversion injuries using an elastic support bandage or an Aircast ankle brace. $\mathrm{Br}$ J Sports Med 2005, 39:9I-96.

6. Adirim TA, Cheng TL: Overview of injuries in the young athlete. Sports Med 2003, 33:75-8I.

7. Viljakka T, Rokkanen P: The treatment of ankle sprain by bandaging and antiphlogistic drugs. Ann Chir Gynaecol 1983, 72:66-70.

8. Nilsson S: Sprains of the lateral ankle ligaments. An epidemiological and clinical study with special reference to different forms of conservative treatment. Part I. Epidemiological and clinical considerations. J Oslo City Hosp 1982, 32:3-29.

9. Garrick JG: The frequency of injury, mechanism of injury, and epidemiology of ankle sprains. Am J Sports Med 1977, 5:24I-242

10. Williams RL, Haddad FS: Comparison of two conservative methods of treating an isolated fracture of the lateral malleolus. J Bone Joint Surg Br 1997, 79:339-340.

II. Rudert M, Wulker N, Wirth C]: [Fibular ligament rupture-conservative or surgical treatment?]. Z Orthop Ihre Grenzgeb 1997, 135:Oa25-Oa26.

12. Povacz P, Unger SF, Miller WK, Tockner R, Resch H: A randomized, prospective study of operative and non-operative treatment of injuries of the fibular collateral ligaments of the ankle. J Bone Joint Surg Am 1998, 80:345-35I.

13. Lynch SA, Renstrom PA: Treatment of acute lateral ankle ligament rupture in the athlete. Conservative versus surgical treatment. Sports Med 1999, 27:6I-7I.

14. Kerkhoffs GM, Handoll HH, de Bie R, Rowe BH, Struijs PA: Surgical versus conservative treatment for acute injuries of the lateral ligament complex of the ankle in adults. Cochrane Database Syst Rev 2002:CD000380.

15. Kerkhoffs GM, Rowe BH, Assendelft WJ, Kelly K, Struijs PA, van Dijk $\mathrm{CN}$ : Immobilisation and functional treatment for acute lateral ankle ligament injuries in adults. Cochrane Database Syst Rev 2002:CD003762.

16. Kannus P, Renstrom P: Treatment for acute tears of the lateral ligaments of the ankle. Operation, cast, or early controlled mobilization. J Bone Joint Surg Am 1991, 73:305-312.

17. Balduini FC, Vegso JJ, Torg JS, Torg E: Management and rehabilitation of ligamentous injuries to the ankle. Sports Med 1987 4:364-380

18. Sommer HM, Schreiber $\mathrm{H}$ : [Early functional conservative therapy of fresh fibular capsular ligament rupture from the socioeconomic viewpoint]. Sportverletz Sportschaden 1993, 7:40-46.

19. Kerkhoffs GM, Struijs PA, Marti RK, Assendelft WJ, Blankevoort L, van Dijk $C N$ : Different functional treatment strategies for acute lateral ankle ligament injuries in adults. Cochrane Database Syst Rev 2002:CD002938.

20. Leanderson J, Wredmark T: Treatment of acute ankle sprain. Comparison of a semi-rigid ankle brace and compression bandage in 73 patients. Acta Orthop Scand 1995, 66:529-531.

21. Eggli S, Sclabas GM, Eggli S, Zimmermann H, Exadaktylos AK: The Bernese ankle rules: a fast, reliable test after low-energy, supination-type malleolar and midfoot trauma. J Trauma 2005, 59:|268-|27|.

22. Hutchison B, Østbye T, Barnsley J, Stewart M, Mathews M, Campbell MK, Vayda E, Harris SB, Torrance-Rynard V, Tyrrell C, Ontario Walk-In Clinic Study: Patient satisfaction and quality of care in walk-in clinics, family practices and emergency departments: the Ontario Walk-In Clinic Study. CMAJ 2003, 168:977-983.

23. Campbell MK, Silver RW, Hoch JS, Østbye T, Stewart M, Barnsley J Hutchison B, Mathews M, Tyrrell C: Re-utilization outcomes and costs of minor acute illness treated at family physician offices, walk-in clinics, and emergency departments. Can Fam Physician 2005, 5 1:82-3.

24. Coker TP: Sport injuries to the foot and ankle. In: Jahss MV, ed. In Disorders of the foot \& ankle - medical and surgical management Philadelphia: WB Saunders Company; 1991:2415-2445.

25. Gauffin $H$, Tropp $H$, Odenrick P: Effect of ankle disk training on postural control in patients with functional instability of the ankle joint. Int J Sports Med 1988, 9:|4|-| 44
26. Freeman MA: Treatment of ruptures of the lateral ligament of the ankle. J Bone Joint Surg Br 1965, 47:66I-668.

27. Perneger TV, Etter JF, Raetzo MA, Schaller P, Stalder H: Comparison of patient satisfaction with ambulatory visits in competing health care delivery settings in Geneva, Switzerland. J Epidemiol Community Health 1996, 50:463-468.

\section{Pre-publication history}

The pre-publication history for this paper can be accessed here:

http://www.biomedcentral.com/1471-2296/9/69/prepub
Publish with Bio Med Central and every scientist can read your work free of charge

"BioMed Central will be the most significant development for disseminating the results of biomedical research in our lifetime. "

Sir Paul Nurse, Cancer Research UK

Your research papers will be:

- available free of charge to the entire biomedical community

- peer reviewed and published immediately upon acceptance

- cited in PubMed and archived on PubMed Central

- yours - you keep the copyright 